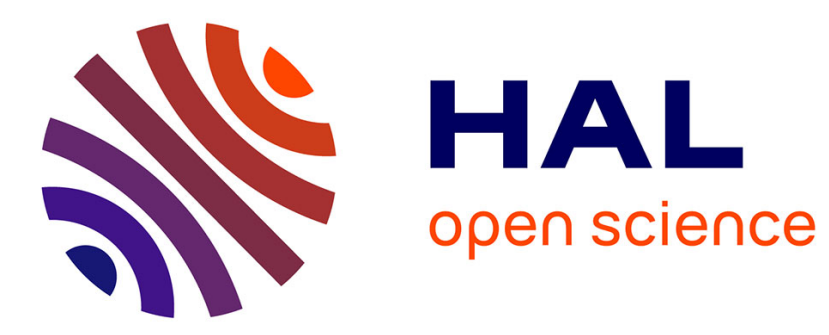

\title{
A reduction of the saddle vertical force triggers the sit-stand transition in cycling
}

Antony Costes, Nicolas A Turpin, David Villeger, Pierre Moretto, Bruno

Watier

\section{- To cite this version:}

Antony Costes, Nicolas A Turpin, David Villeger, Pierre Moretto, Bruno Watier. A reduction of the saddle vertical force triggers the sit-stand transition in cycling. Journal of Biomechanics, 2015, 48 (12), pp.2998-3003. 10.1016/j.jbiomech.2015.07.035 . hal-01663013

\section{HAL Id: hal-01663013 https://hal.laas.fr/hal-01663013}

Submitted on 13 Dec 2017

HAL is a multi-disciplinary open access archive for the deposit and dissemination of scientific research documents, whether they are published or not. The documents may come from teaching and research institutions in France or abroad, or from public or private research centers.
L'archive ouverte pluridisciplinaire HAL, est destinée au dépôt et à la diffusion de documents scientifiques de niveau recherche, publiés ou non, émanant des établissements d'enseignement et de recherche français ou étrangers, des laboratoires publics ou privés. 
ANTONY COSTES ${ }^{\mathrm{a}, *}$

$10{ }^{a}$ University of Toulouse, UPS, PRISSMH, 118 Route de Narbonne, 31062 Toulouse Cedex 9, France

$11{ }^{b}$ Center for Interdisciplinary Research in Rehabilitation (CRIR), Institut de Réadaptation Gingras-

12 Lindsay de Montréal and Jewish Rehabilitation Hospital, Laval, Quebec, Canada

$13{ }^{c}$ University of Toulouse; UPS; CRCA; 118 route de Narbonne, F-31062 Toulouse Cedex 9, France

$14{ }^{d}$ CNRS; CRCA; 118 route de Narbonne, F-31062 Toulouse Cedex 9, France

$15{ }^{e}$ CNRS, LAAS, 7 Avenue du Colonel Roche, F-31400 Toulouse, France

$16{ }^{f}$ University of Toulouse, UPS, LAAS, F-31400 Toulouse, France

$18{ }^{*}$ Corresponding author. Tel.: +33 (0) 5615564 40; Fax: +33 (0) 561558280

19 E-mail address: antony.costes@univ-tlse3.fr (A. Costes).

21 Running Title: "Triggers of the Sit-Stand Transition in Cycling"

22 Word count: 3576 (abstract: 235). 
25 The purpose of the study was to establish the link between the saddle vertical force and its

26 determinants in order to establish the strategies that could trigger the sit-stand transition. We

27 hypothesized that the minimum saddle vertical force would be a critical parameter influencing the 28 sit-stand transition during cycling. Twenty-five non-cyclists were asked to pedal at six different 29 power outputs from $20 \%\left(1.6 \pm 0.3 \mathrm{~W} \cdot \mathrm{kg}^{-1}\right)$ to $120 \%\left(9.6 \pm 1.6 \mathrm{~W} \cdot \mathrm{kg}^{-1}\right)$ of their spontaneous sit-stand 30 transition power obtained at 90RPM. Five 6-components sensors (saddle tube, pedals and 31 handlebars) and a full-body kinematic reconstruction were used to provide the saddle vertical force 32 and other force components (trunk inertial force, hips and shoulders reaction forces, and trunk 33 weight) linked to the saddle vertical force. Minimum saddle vertical force linearly decreased with 34 power output by $87 \%$ from a static position on the bicycle $\left(5.30 \pm 0.50 \mathrm{~N} . \mathrm{kg}^{-1}\right)$ to power 35 output $=120 \%$ of the sit-stand transition power $\left(0.68 \pm 0.49 \mathrm{~N} \cdot \mathrm{kg}^{-1}\right)$. This decrease was mainly 36 explained by the increase in pedal forces from $2.84 \pm 0.58 \mathrm{~N} \cdot \mathrm{kg}^{-1}$ to $6.57 \pm 1.02 \mathrm{~N} . \mathrm{kg}^{-1}$ from 20 to $37120 \%$ of the power output corresponding to the sit-stand transition, causing an increase in hip 38 vertical forces from $-0.17 \mathrm{~N} \cdot \mathrm{kg}^{-1}$ to $3.29 \mathrm{~N} \cdot \mathrm{kg}^{-1}$. The emergence of strategies aiming at counteracting 39 the elevation of the trunk (handlebars and pedals pulling) coincided with the spontaneous sit-stand 40 transition power. The present data suggest that the large decrease in minimum saddle vertical force 41 observed at high pedal reaction forces might trigger the sit-stand transition in cycling. 


\section{Introduction}

Seated (SEAT) and Standing (STAND) are the two common positions chosen during bicycle

47 locomotion. Several studies comparing the two positions have shown that spontaneous pedaling cadences are slower in STAND than in SEAT position (Harnish et al., 2007; Lucía et al., 2001), and that the STAND position is associated with the highest power outputs (McLester et al., 2004; Millet et al., 2002; Reiser et al., 2002). Furthermore, the fact that cyclists tend to spontaneously switch

51 from SEAT to STAND when high force applied to the pedals are needed (i.e. during fast 52 accelerations or steep climb ascensions) suggests that the change in position favors a maximization of the pedal reaction forces (Hansen and Waldeland, 2008). However, the parameters leading to select one position over the other one in order to produce a given combination of pedal reaction

55 force and power output need to be clarified.

Many attempts have been made to understand the mechanisms underlying these positions, particularly to determine the superiority of the STAND position to produce higher power outputs and pedal reaction forces. From a joint torque perspective, a study using the moment cost function defined by Gonzalez and Hull (1989) presented a slight reduction of this cost function above the sitstand transition power (Poirier et al., 2007), whereas lower limbs net joint torques have been described by others as increasing in STAND position for both the ankle plantarflexion and the knee extension (Caldwell et al., 1999; Li and Caldwell, 1998). From a metabolic energy consumption perspective, the SEAT position has been shown to be more efficient to produce lower power outputs (Ryschon and Stray-Gundersen, 1991; Tanaka et al., 1996), and equally efficient as the STAND one to produce high power outputs (Harnish et al., 2007; Millet et al., 2002; Tanaka et al., 1996). Regarding studies using electromyography, the literature suggests that differences in the temporal profiles and in the level of activation of the muscles could be expected between SEAT and STAND

68 (Li and Caldwell, 1998; Hug et al., 2011). For example, Duc et al. (2008) reported a slight decrease 
69 for the semimembranosus activation from SEAT to STAND, whereas Li and Caldwell (1998)

70 reported increased activations of the gluteus maximus, tibialis anterior and rectus femoris muscles

71 in STAND position. These differences may influence the coordination patterns in both positions (De

72 Marchis et al., 2013). Nonetheless, the muscle synergies activated in the two positions may remain

73 similar (Hug et al., 2011) and the literature does not provide evidences of an advantage of one

74 position against the other at this level.

Since there is no obvious reason to prefer the STAND rather than the SEAT position to

76 produce one given power output, we propose in this study to reverse the questioning and to wonder

77 why the SEAT position is no longer optimal, instead of why the STAND position becomes optimal

78 beyond a given level of crank power. To test our hypotheses, we first propose a criterion that could

79 clearly distinguish the two positions: the SEAT position is characterized by a contact between the

80 cyclist and the saddle (i.e. a vertical force is applied by the cyclist on the saddle) whereas the

81 STAND position is characterized by the absence of this vertical force. In this definition, the force

82 applied by the cyclist on the saddle (and reciprocally) is of central interest, and the sit-stand

83 transition is defined by the disappearance of this force. To the best of our knowledge, only three

84 studies measured saddle forces in cycling. The first one presented saddle force at three pedaling

85 cadences and described a double period pattern with maximum magnitudes decreasing as cadence

86 decreases (Bolourchi and Hull, 1985). However, the second study, did not found this double period

87 pattern (Stone and Hull, 1995) while the third one observed both of these patterns (Wilson and

88 Bush, 2007). To better understand this phenomenon, we propose to investigate the saddle force

89 patterns. According to Newton's second law, this force is the result of a simple mechanical

90 interaction between the cyclist's body weight and the other forces applied on his bicycle.

91 Consequently, a downward vertical force applied on the pedal would result by reaction in an upward

92 force on the hip, accelerating the trunk in an upward direction, and decreasing the force applied on

93 the saddle by the cyclist. Therefore, we propose to measure vertical forces applied on the saddle, in 
94 complement with the other forces acting on the trunk of the cyclist (i.e. hips and shoulders reaction

95 forces, trunk weight, and acceleration of the trunk's center-of-mass) at different pedal reaction

96 forces. The aims of this study are to validate a full-body inverse dynamics model of cycling and to

97 test the hypothesis that saddle vertical force would decrease and reach values close to zero with

98 increasing pedal forces, making the SEAT position irrelevant given its definition and leading the

99 cyclist to spontaneously adopt the STAND position.

100

101

2. Methods

102

\subsection{Participants}

104

Twenty five male sport science students $(23.2 \pm 3.6 \mathrm{y}$, height $1.77 \pm 0.06 \mathrm{~m}$, body mass

$71.5 \pm 9.1 \mathrm{~kg}$ ) volunteered for this investigation. The participants were non-cyclists and belonged to category 4-5 according to Ansley and Cangley (2009) classification. Each participant was informed of the experimental procedure and signed an informed consent form prior to the study. The study was conducted in accordance with the declaration of Helsinki and was approved by the University of Toulouse ethical committee. Participants were asked to avoid high-intensity or exhaustive

111 exercise at least 72 hours before the laboratory trials.

\subsection{Experimental Protocol}

The cycling tests were performed using an electromagnetically braked cycle ergometer

116 Excalibur (LODE, Groningen, Netherlands). To limit bike positioning effects, standardized settings

117 were adopted. Briefly, pedal cleats were positioned under the first metatarsal bone (Viker and

118 Richardson, 2013), the saddle height was set at a $150^{\circ}$ knee angle during maximum leg extension, 
119 the seat tube angle was set to $73^{\circ}$, the crank length was $0.17 \mathrm{~m}$ in length and the handlebar was flat.

120 The latter was positioned to standardize drop (the vertical distance between the top of the saddle

121 and the handlebar mediolateral axis) and reach (the horizontal distance between the back of the

122 saddle and the handlebar mediolateral axis) lengths according to torso and arm lengths (de Vey

123 Mestdagh, 1998). The mediolateral positioning of the two hands on the handlebar was left up to the

124 participant (handlebar width: $0.7 \mathrm{~m}$ ).

125 After bike positioning, participants were first weighed on the cycle ergometer in order to

126 measure a static level of saddle vertical force (representing $0 \%$ of the sit-stand transition power).

127 This weighing was made with the shoes fixed on the pedals, the hands on the handlebars, and the

128 cranks in horizontal position. Then, after a five-minute warm-up at $100 \mathrm{~W}$, they performed a cycling

129 test to determine their spontaneous sit-stand transition power (Figure 1). In this test, phases of $20 \mathrm{~s}$

130 with a starting power output of $200 \mathrm{~W}$ incremented by $25 \mathrm{~W}$ at each step rest were alternated with

131 rest phases of $40 \mathrm{~s}$ at a power output of $50 \mathrm{~W}$. The sit-stand transition power was considered as the

132 power output at which participants rose from the saddle during at least $10 \mathrm{~s}$. A visual feedback of

133 the pedaling cadence was provided to the participants who were instructed to maintain it at $13490 \pm 5$ RPM.

135

PLEASE INSERT FIGURE 1

Then, after a five-minute rest period, participants performed six randomized trials at power 139 output corresponding to $20,40,60,80,100$ or $120 \%$ of their sit-stand transition power and were 140 asked to remain seated throughout these sequences. Each pedaling trial began with a minimum 141 stabilization time of $10 \mathrm{~s}$ at the target power output at 90RPM, followed by $10 \mathrm{~s}$ of data recording. 142 Three minutes of passive rest were given between each of these six trials. 
The 3D force and moment components applied to the handlebar, saddle tube and pedals were recorded from three tubular sensors (SENSIX, Poitiers, France), and by two instrumented pedals (Idynamometers had a maximum $1 \%$ error on each direction (combining linearity and hysteresis errors), and a maximum $1.5 \%$ error on the 6 components combination.

PLEASE INSERT FIGURE 2 cameras (VICON, Oxford, United-Kingdom) at $200 \mathrm{~Hz}$. The kinetics sensors' reference points were defined as shown in Figure 2. The ankle (because of the impossibility to stick one kinematic marker on the medial malleollus in reason of the crank proximity), shoulder and hip joint centers were located using the SCoRE method (Ehrig et al., 2006). For this method, a preliminary recording asking the participants to repeat flexion-extension, abduction-adduction and circumduction of the tested joint allowed the localization of their centers-of-rotation (Begon et al., 2007). Body segments masses, center-of-mass positions, and radii of gyration were defined in accordance with De Leva's anthropometric charts (de Leva, 1996). All kinetics and kinematics data were recorded in threedimensions.

\subsection{Data reduction and analysis}

Kinetics and kinematics data were synchronized using Nexus 1.7.1 system (VICON, Oxford,

168 United-Kingdom) and filtered using a $4^{\text {th }}$ order, zero phase-shift, low-pass Butterworth with a $8 \mathrm{~Hz}$ 
cutoff frequency (McDaniel et al., 2014). In order to determine the factors affecting the saddle

170 vertical force, the trunk was represented (comprising the head and the pelvis) as being submitted to 171 external forces applied on the shoulders, hips, and saddle contact. The following equality has been 172 computed by isolating the head and trunk solid according to Newton's second law:

$174 \quad F_{s}=m_{t} a_{t}-\left(W_{t}+F_{h}+F_{s h}\right) \quad($ Equation 1$)$

where $\mathrm{m}_{\mathrm{t}}$ is the mass of the head and trunk solid according to De Leva's anthropometric chart, $a_{t}$ is the linear acceleration of the head and trunk center-of-mass, $W_{t}$ is the sum of the head and trunk weights, $F_{s}$ is the saddle reaction force obtained from the saddle tube sensor, $F_{s h}$ is the shoulder reaction force calculated by inverse dynamics method from the handlebar sensors, and $\mathrm{F}_{\mathrm{h}}$

180 the hip reaction force calculated by inverse dynamics method from the pedal sensors. To compute $181 \mathrm{~F}_{\mathrm{h}}$ and $\mathrm{F}_{\mathrm{sh}}$, a classic inverse dynamic process was used (Winter, 1990). In this method, body182 segments from upper and lower limbs were considered rigid and interconnected by frictionless 183 joints and their inertial parameters were derived from the scaling equations (de Leva, 1996). Given 184 the aims of the study, only the vertical components in Equation 1 were considered. This model is 185 illustrated in Figure 3. The entire data processing was performed using custom-made codes written in Scilab 5.4.0 (SCILAB, Scilab Enterprises). All the data were normalized to the subject's body mass. During the crank cycle corresponding to the minimum saddle vertical force observed among the $10 \mathrm{~s}$ of recording for each power output, vertical forces presented in Equation 1 were extracted.

189 In this crank cycle and at the instant corresponding to the minimum saddle vertical force, vertical 190 force values were retained for further analyses. 
Before each statistical test, data normality and variance homogeneity were assessed using

197 Shapiro-Wilk's, and Levene's tests, respectively. A one-way repeated measures ANOVA (power output $=20,40,60,80,100$ and $120 \%$ of sit-stand transition power) was performed to compare saddle force levels across Power outputs. Post-hoc analyses were performed using Bonferroni's method. To check the accuracy of the experimental model represented by the equality computed in

201 Equation 1, the difference between saddle vertical reaction force and the equivalent sum of forces was quantified for each power output condition using the root-mean-square error (RMSE). In addition, Pearson's coefficients $(\mathrm{R})$ were used to determine the correlation between the two patterns. Partial eta-squared $\left(\eta^{2}\right)$ was used to quantify the size of the effect of power output on vertical forces. All statistical analyses were performed using STATISTICA (STATSOFT, MaisonsAlfort, France). A p-value of 0.05 was defined as the level of statistical significance.

\section{Results}

The sit-stand transition power reached during a pedalling phase of $20 \mathrm{~s}$ at 90 RPM (i.e.

211 during the first test, see methods) was $568 \pm 93 \mathrm{~W}\left(8.0 \pm 1.4 \mathrm{~W} \cdot \mathrm{kg}^{-1}\right)$ and the power outputs 212 corresponding to $20,40,60,80,100$ and $120 \%$ of sit-stand transition power were $114 \pm 19 \mathrm{~W}$ $213\left(1.6 \pm 0.3 \mathrm{~W}^{\mathrm{kg}}{ }^{-1}\right), 227 \pm 37 \mathrm{~W}\left(3.2 \pm 0.5 \mathrm{~W}^{\mathrm{k}} \mathrm{kg}^{-1}\right), 341 \pm 56 \mathrm{~W}\left(4.8 \pm 0.8 \mathrm{~W} \cdot \mathrm{kg}^{-1}\right), 454 \pm 74 \mathrm{~W}$ $214\left(6.4 \pm 1.1 \mathrm{~W} \cdot \mathrm{kg}^{-1}\right), 568 \pm 93 \mathrm{~W}\left(8.0 \pm 1.4 \mathrm{~W} \cdot \mathrm{kg}^{-1}\right)$ and $682 \pm 111 \mathrm{~W}\left(9.6 \pm 1.6 \mathrm{~W} \cdot \mathrm{kg}^{-1}\right)$, respectively.

215 The static vertical force on the saddle $(0 \%$ of sit-stand transition power) was $5.30 \pm 0.50$ 216 N.kg-1.

Descriptive statistics about saddle vertical force are shown in Table 1. A significant main 218 effect $(p<0.001)$ of power output was found, showing that the magnitudes of minimum saddle 
219 vertical forces decreased with increasing power output. Post-hoc tests indicated that the saddle

220 vertical force decreased significantly between each power output condition.

221

PLEASE INSERT TABLE 1

223

Accuracy of the model was assessed and the results of the saddle vertical force pattern

reconstruction using the equality described in Equation 1 are presented in Table 2. An illustration of

226 this reconstruction is presented in Figure 4.

Vertical saddle, trunk inertial force, shoulders and hips reaction force patterns are presented

232 in Figure 5.

237 minimum saddle vertical force in the cycle is presented in Figure 6. 
244 forces would decrease with increasing power output. Our findings supported our hypothesis with a

245 linear decrease of $87.4 \%$ of the saddle vertical reaction force, from $5.30 \pm 0.50 \mathrm{~N} . \mathrm{kg}^{-1}$ to $0.68 \pm 0.49$

$246 \mathrm{~N} . \mathrm{kg}^{-1}$, between a static position on the bicycle and the minimum instantaneous value obtained

247 while pedaling at $120 \%$ of the sit-stand transition power (Table 1). Another purpose of the study

248 was to determine the forces applied on the trunk during cycling at different pedal reaction forces in

249 order to interpret the decrease in saddle vertical force. The model presented in Equation 1 provided

250 an accurate examination of the forces associated with the saddle vertical force (Table 2 and Figure

251 4). These data suggest that the vertical saddle force decreased mainly in response to the increase in

252 hip vertical reaction forces (Figures 5 and 6). Consequently, with increasing pedal reaction forces,

253 the body weight was less and less supported by the saddle. The results indicated that when the

254 saddle force approached $1 \mathrm{~N} . \mathrm{kg}^{-1}$, the participants tended to spontaneously transit to the STAND

255 position, suggesting that the saddle force could be a predictor of the sit-stand transition power.

A combination of several strategies was observed to limit the decrease in saddle vertical

force in response to the increasing demand in pedal force, potentially increasing both the sit-stand transition power and the delay before the occurrence of the sit-stand transition. These strategies are

likely to help maintaining the SEAT position when high level of pedal reaction forces are created and may also explain why the saddle vertical force did not reach zero (Figure 6). However, these strategies have been previously reported as particularly metabolically costly (Korff et al., 2007;

262 Edwards et al., 2009; McDaniel et al., 2005). The first strategy observed was to pull on the pedal to

263 create downward reaction forces at the hip level (Figure 5). This pedal pulling may be associated

264 with the advantage of increasing the mechanical effectiveness of pedaling (Korff et al. 2007), and 265 explains the non-linear increase in the sum of pedal vertical forces during with increasing crank 266 power (Figure 6). However, and probably because human's lower limb is far stronger to produce 267 force in extension than in flexion (Anderson et al., 2007), increasing the mechanical effectiveness 
268 by training cyclists to pull more on the pedals has been reported to decrease their metabolic 269 efficiency (Korff et al., 2007; Edwards et al., 2009). Because experts in cycling have been reported 270 to push more on the pedals at equivalent power output (Coyle et al., 1991) it could be expected that 271 they would have to create downward forces by pulling their handlebars and/or pedals and/or 272 accelerating their trunk downward simultaneously to the decrease in vertical saddle force at lower 273 power outputs than the non-cyclists from our study, and more frequently in their daily practice 274 because of the higher power output that they develop. Further investigations are needed to confirm 275 this hypothesis which could lead to improvement in cycling performance. A second strategy 276 observed to limit the reduction of the saddle vertical force was to accelerate the trunk's center-ofmass downward (Figure 5). It is worth noting that the pattern of these accelerations are synchronized with the pattern of saddle vertical force from $100 \%$ of the sit-stand transition power: when the saddle force was at its minimum, the trunk's center-of-mass was accelerated downward, 280 and reciprocally, the upward acceleration of the trunk's center-of-mass occurred while the saddle 281 vertical force was at its maximum, the whole occurring twice by pedaling cycle. A third strategy was to create a downward reaction force at the shoulders by pulling on the handlebar, this last strategy was mainly observed above the sit-stand transition power (Figure 6). Both of these 284 strategies involve additional muscular efforts from the upper limbs. As highlighted by McDaniel et 285 al. (2005), the upper limbs' metabolic cost is important in cycling. These authors showed that the 286 use of a modified saddle allowing the stabilization of the trunk and a potential decrease in upper 287 limb muscular efforts decreased the metabolic cost of pedaling for a fixed power output. The 288 reductions were of $1.6,1.2$, and $0.2 \%$ at 40,60 , and 80 RPM, respectively and they showed that the 289 best improvement in metabolic cost was obtained at the highest level of pedal forces (for a fixed 290 power output), i.e. in the conditions corresponding to the highest handlebars and pedals pulling and 291 trunk inertial forces observed in our study. The present data are in agreement with the interpretation 292 that with increasing pedal forces, the body weight was less and less supported by the saddle, and 
293 that downward forces acting on the trunk were required to maintain the SEAT position above one

294 level of crank power (for a given pedaling cadence of 90 RPM). The fact that costly strategies to

295 counteract the elevation of the trunk emerged at the power at which the participants spontaneously

296 switched to the STAND position suggests that this position could have been chosen in order to

297 avoid these strategies. It is worth mentioning that several other factors may influence the choice of

298 the cycling position in the field such as aerodynamics (Debraux et al., 2011; Millet et al., 2014), or

299 slope gradient (Bertucci et al., 2005; Duc et al., 2008). However, the difficulty to keep force on the

300 saddle during high pedal reaction force production observed in this study is making the SEAT

301 position less attractive in these conditions, giving a mechanical reason to trigger the sit-stand

302 transition. Our study is the first to present saddle force patterns at different levels of pedal reaction

303 force as a justification to trigger the sit-stand transition, and to explain these patterns by a

304 mechanical decomposition of the forces applied on the trunk during cycling. In order to further

305 confirm the present results, experimental designs manipulating the body weight, and/or testing

306 pedaling cadence effects on the magnitude of saddle vertical force and the occurrence of the sit-

307 stand transition are warranted. Additionally, Hansen and Waldeland (2008) implemented repeated

308 cycling bouts to exhaustion with experimented cyclists and reported smaller sit-stand transition

309 power output than the one observed in this study with non-cyclists. This difference illustrates a

310 potential protocol-dependence of the sit-stand transition power, which may therefore also be

311 affected by the duration of the cycling trial. Altogether, further investigations on the sit-stand

312 transition paradigm in cycling may lead to improvements in pedaling efficiency by potentially

313 decreasing the mechanical cost of pedaling in SEAT position at high pedal reaction forces, and by

314 determining the precise pedal reaction force level at which the sit-stand transition is necessary to

315 maximize performance for different cadences, weights and durations conditions.

By determining the parameters involved in saddle force patterns, the present study also have

317 implication for clinicians, researchers, and manufacturers trying to understand the etiology of groin 
318 injuries and erectile dysfunction associated with cycling (Bressel et al., 2010; Bressel and Larson,

319 2003; Carpes et al., 2009; Lowe et al., 2004). Indeed, the inconsistency of the patterns of saddle

320 force observed previously (Bolourchi and Hull, 1985; Stone and Hull, 1995; Wilson and Bush,

321 2007) can be explained by the different pedaling conditions used in these studies. Due to the

322 sensitiveness of saddle forces (and thus saddle pressures) to pedal reaction forces, cyclists suffering

323 from these pathologies should decrease their pedaling cadence for the same workload, as this is

324 supposed to increase hip upward reaction force in order to decrease the saddle reaction force.

It is important to note some limitations of the present study. The use of a cycling ergometer

326 is a common practice for testing, rehabilitation and training, but it differs with cycling in the field

327 (Bertucci et al., 2012). Likewise, the potential protocol-dependence of the spontaneous sit-stand 328 transition power determination needs further investigations.

\section{Conclusion}

332 The body weight is gradually less supported by the saddle as pedal reaction forces increase, thus

333 decreasing the mechanical advantage of pedaling in the SEAT position. Strategies counteracting the

334 upward vertical pedal forces were observed around the power corresponding to the sit-stand 335 transition, suggesting that the spontaneous choice to rise in the STAND position may be a solution 336 to reduce the need to overcome these constraints. The spontaneous sit-stand transition occurred at 337 minimum saddle vertical force about $1 \mathrm{~N} \cdot \mathrm{kg}^{-1}$; the high linearity of the relationship between saddle 338 vertical force and power output for a given cadence suggesting an ability of prediction of the sit339 stand transition.

\section{Acknowledgements}


343 Antony Costes was funded by a PhD grant from the French Ministry of Education and Research

344 (Ministère de 1'Education et de la Recherche). The authors would like to thank Dr. Laurent Seitz for 345 his review of the manuscript.

346

347 Conflict of Interest

348

349 The authors have no financial or personal relationships with other people or organizations that could 350 have inappropriately influenced this research. 


\section{References}

353 Anderson, D.E., Madigan, M.L., Nussbaum, M.A., 2007. Maximum voluntary joint torque as a

354 function of joint angle and angular velocity: Model development and application to the lower limb.

355 J. Biomech. 40, 3105-3113.

357 Ansley, L., Cangley, P., 2009. Determinants of “optimal” cadence during cycling. Eur. J. Sport Sci. $3589,61-85$.

360 Begon, M., Monnet, T., Lacouture, P., 2007. Effects of movement for estimating the hip joint centre. 361 Gait Posture 25, 353-359.

Bertucci, W., Betik, A., Duc, S., Grappe, F., 2012. Gross Efficiency and Cycling Economy Are 644.

367 Bertucci, W., Grappe, F., Girard, A., Betik, A., Rouillon, J.D., 2005. Effects on the crank torque 368 profile when changing pedalling cadence in level ground and uphill road cycling. J. Biomech. 38, $369 \quad 1003-1010$.

371 Bini, R., Hume, P.A., Croft, J.L., 2011. Effects of bicycle saddle height on knee injury risk and 372 cycling performance. Sports Med. 41, 463-476.

374 Bolourchi, F., Hull, M.A., 1985. Measurement of Rider Induced Loads During Simulated Bicycling.

375 Int. J. Sports Biomech. 1, 308-329. 
377 Bressel, E., Larson, B.J., 2003. Bicycle seat designs and their effect on pelvic angle, trunk angle,

378 and comfort. Med. Sci. Sports Exerc. 35, 327-332.

379

380 Bressel, E., Nash, D., Dolny, D., 2010. Association between Attributes of a Cyclist and Bicycle Seat 381 Pressure. J. Sex. Med. 7, 3424-3433.

383 Caldwell, G.E., Hagberg, J.M., McCole, S.D., Li, L., 1999. Lower Extremity Joint Moments During 384 Uphill Cycling. J. Appl. Biomech. 15, 166-181.

386 Carpes, F.P., Dagnese, F., Kleinpaul, J.F., Martins, E. de A., Mota, C.B., 2009. Bicycle Saddle 387 Pressure: Effects of Trunk Position and Saddle Design on Healthy Subjects. Urol. Int. 82, 8-11.

389 Coyle, E.F., Feltner, M.E., Kautz, S.A., Hamilton, M.T., Montain, S.J., Baylor, A.M., Abraham, 390 L.D., Petrek, G.W., 1991. Physiological and biomechanical factors associated with elite endurance 391 cycling performance. Med. Sci. Sports Exerc. 23, 93-107.

393 Debraux, P., Grappe, F., Manolova, A.V., Bertucci, W., 2011. Aerodynamic drag in cycling: methods 394 of assessment. Sports Biomech. 10, 197-218.

396 De Marchis, C., Schmid, M., Bibbo, D., Castronovo, A.M., D’Alessio, T., Conforto, S., 2013. 397 Feedback of mechanical effectiveness induces adaptations in motor modules during cycling. Front. 398 Comput. Neurosci. 17, 7-35.

400 De Leva, P., 1996. Adjustments to Zatsiorsky-Seluyanov's segment inertia parameters. J. Biomech. 
403 De Vey Mestdagh, K., 1998. Personal perspective: in search of an optimum cycling posture. Appl. 404 Ergon. 29, 325-334.

Duc, S., Bertucci, W., Pernin, J.N., Grappe, F., 2008. Muscular activity during uphill cycling: effect 407 of slope, posture, hand grip position and constrained bicycle lateral sways. J. Electromyogr. 408 Kinesiol. 18, 116-127.

410 Edwards, L.M., Jobson, S.A., George, S.R., Day, S.H., Nevill, A.M., 2009. Whole-body efficiency 411 is negatively correlated with minimum torque per duty cycle in trained cyclists. J. Sports Sci. 27, $412 \quad 319-325$.

413

414 Ehrig, R.M., Taylor, W.R., Duda, G.N., Heller, M.O., 2006. A survey of formal methods for 415 determining the centre of rotation of ball joints. J. Biomech. 39, 2798-2809.

417 Gonzalez, H., Hull, M.L., 1989. Multivariable optimization of cycling biomechanics. J. Biomech. 418 22, 1151-1161.

420 Hansen, E.A., Waldeland, H., 2008. Seated versus standing position for maximization of 421 performance during intense uphill cycling. J. Sports Sci. 26, 977-984.

423 Harnish, C., King, D., Swensen, T., 2007. Effect of cycling position on oxygen uptake and preferred 424 cadence in trained cyclists during hill climbing at various power outputs. Eur. J. Appl. Physiol. 99, $425 \quad 387-391$. 
427 Hug, F., Turpin, N.A., Couturier, A., Dorel, S., 2011. Consistency of muscle synergies during

428 pedaling across different mechanical constraints. J. Neurophysiol. 106, 91-103.

429

430 Korff, T., Romer, L.M., Mayhew, I., Martin, J.C., 2007. Effect of pedaling technique on mechanical 431 effectiveness and efficiency in cyclists. Med. Sci. Sports Exerc. 39, 991-995.

433 Li, L., Caldwell, G.E., 1998. Muscle coordination in cycling: effect of surface incline and posture.

434 J. Appl. Physiol. 85, 927-934.

436 Lowe, B.D., Schrader, S.M., Breitenstein, M.J., 2004. Effect of bicycle saddle designs on the 437 pressure to the perineum of the bicyclist. Med. Sci. Sports Exerc. 36, 1055-1062.

Lucía, A., Hoyos, J., Chicharro, J.L., 2001. Preferred pedalling cadence in professional cycling. 440 Med. Sci. Sports Exerc. 33, 1361-1366.

442 McDaniel, J., Behjani, N.S., Elmer, S.J., Brown, N.A., Martin, J.C., 2014. Joint-specific power443 pedaling rate relationships during maximal cycling. J. Appl. Biomech. 30, 423-430.

445 McDaniel, J., Subudhi, A., Martin, J.C., 2005. Torso stabilization reduces the metabolic cost of 446 producing cycling power. Can. J. Appl. Physiol. 30, 433-441.

448 McLester, J.R., Green, J.M., Chouinard, J.L., 2004. Effects of standing vs. seated posture on 449 repeated Wingate performance. J. Strength Cond. Res. 18, 816-820. 
451 Millet, G.P., Tronche, C., Fuster, N., Candau, R., 2002. Level ground and uphill cycling efficiency

452 in seated and standing positions. Med. Sci. Sports Exerc. 34, 1645-1652.

453

454 Millet, G.P., Tronche, C., Grappe, F., 2014. Accuracy of indirect estimation of power output from 455 uphill performance in cycling. Int. J. Sports Physiol. Perform. 9, 777-82.

457 Poirier, E., Do, M., Watier, B., 2007. Transition from seated to standing position in cycling allows 458 joint moment minimization. Sci. Sports 22, 190-195.

460 Reiser, R.F., 2nd, Maines, J.M., Eisenmann, J.C., Wilkinson, J.G., 2002. Standing and seated 461 Wingate protocols in human cycling. A comparison of standard parameters. Eur. J. Appl. Physiol. $46288,152-157$.

464 Ryschon, T.W., Stray-Gundersen, J., 1991. The effect of body position on the energy cost of cycling. 465 Med. Sci. Sports Exerc. 23, 949-953.

467 Stone, C., Hull, M.L., 1995. The effect of rider weight on rider-induced loads during common 468 cycling situations. J. Biomech. 28, 365-375.

470 Tanaka, H., Bassett, D.R., Jr, Best, S.K., Baker, K.R., Jr, 1996. Seated versus standing cycling in 471 competitive road cyclists: uphill climbing and maximal oxygen uptake. Can. J. Appl. Physiol. 21, $472149-154$.

473

474 Viker, T., Richardson, M.X., 2013. Shoe cleat position during cycling and its effect on subsequent 475 running performance in triathletes. J. Sports Sci. 31, 1007-1014. 
477 Wilson, C., Bush, T.R., 2007. Interface forces on the seat during a cycling activity. Clin. Biomech.

$478 \quad 22,1017-1023$.

479

480 Winter, D.A., 1990. Biomechanics and Motor Control of Human Movement. Wiley-Interscience, 481 New York, pp. 370.

482 
FIGURE 1 - Experimental protocol to determine the sit-1 to-stand transition power (SSTP). SSTP was considered as the CPO at which the participants rose from the saddle during at least $10 \mathrm{~s}$.

FIGURE 2 - 3D force and moment sensors. A. Pedal. B. Saddle Tube. C. Handlebars.

FIGURE 3 - Theoretical model of the cyclist. For clarity only one side of the body is represented. Red arrows represent external forces (saddle, pedals and handlebars), and dashed red arrows represent reaction forces applied on the trunk at the hip and shoulder levels calculated by inverse dynamics. Only the vertical components of these forces are represented. White dots represent kinematic markers. Black dots represent joint centers calculated using the SCoRE method.

FIGURE 4 - Illustration of the mean saddle vertical reaction force and mean sum of forces applied on the trunk (presented in Equation 1) patterns for all participants $(n=25)$ for CPO $=20 \%$ of SSTP.

FIGURE 5 - Vertical reaction force patterns presented along the crank cycle corresponding to the minimum saddle vertical reaction force recorded for each CPO. Mean lefts (red line) and rights (blue line) are presented \pm one standard deviation. Data normalized by body-mass. A. Saddle. B. Mass time acceleration of the trunk's center-of-mass. C. Shoulders. D. Hips.

FIGURE 6 - Evolution of the vertical reaction forces across CPOs. Diamonds: saddle vertical reaction forces. Squares: product between the mass of the trunk and the acceleration of its center of mass. Triangles: sum of the two hip vertical reaction forces. White circles: sum of the two shoulder vertical reaction forces. Black dots: weight of the 26 head and trunk. Each data point corresponds to the instantaneous vertical force observed while the saddle vertical force was minimal. Positive values indicate upward reaction forces (except for the trunk's weight, reverted in a purpose of readability). 30

TABLE 1 - Minimum saddle vertical reaction forces across CPOs. Data are expressed in N.kg- as mean \pm standard deviation [range]. *: Main CPO effect.a,b,c,d,e, and f represent significant differences compared to $20,40,60,80,100$, and $120 \%$ of SSTP conditions, respectively $(\mathbf{p}<\mathbf{0 . 0 0 1})$.

TABLE 2 - Accuracy of the mechanical decomposition. Root-Mean-Square-Error (RMSE) expressed in N.kg-1, and coefficients of correlation $(R)$ between the pattern of vertical saddle force and the pattern of the sum of forces applied on the trunk (terms described in Equation 1) are presented as MEAN $( \pm S D)$. * represents significant coefficient of correlation $40(\mathbf{P}<\mathbf{0 . 0 0 1})$. 


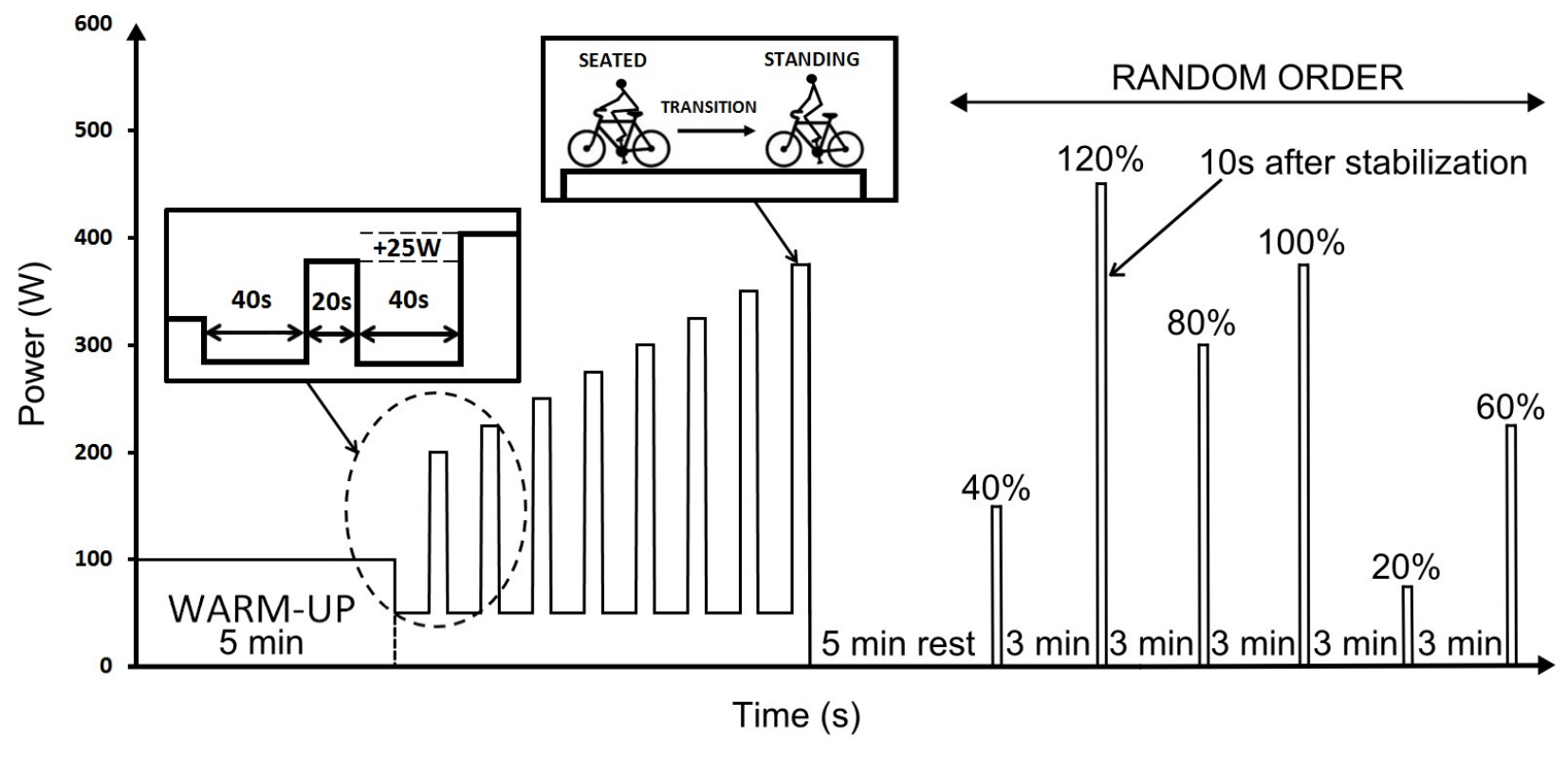

FIGURE 1 


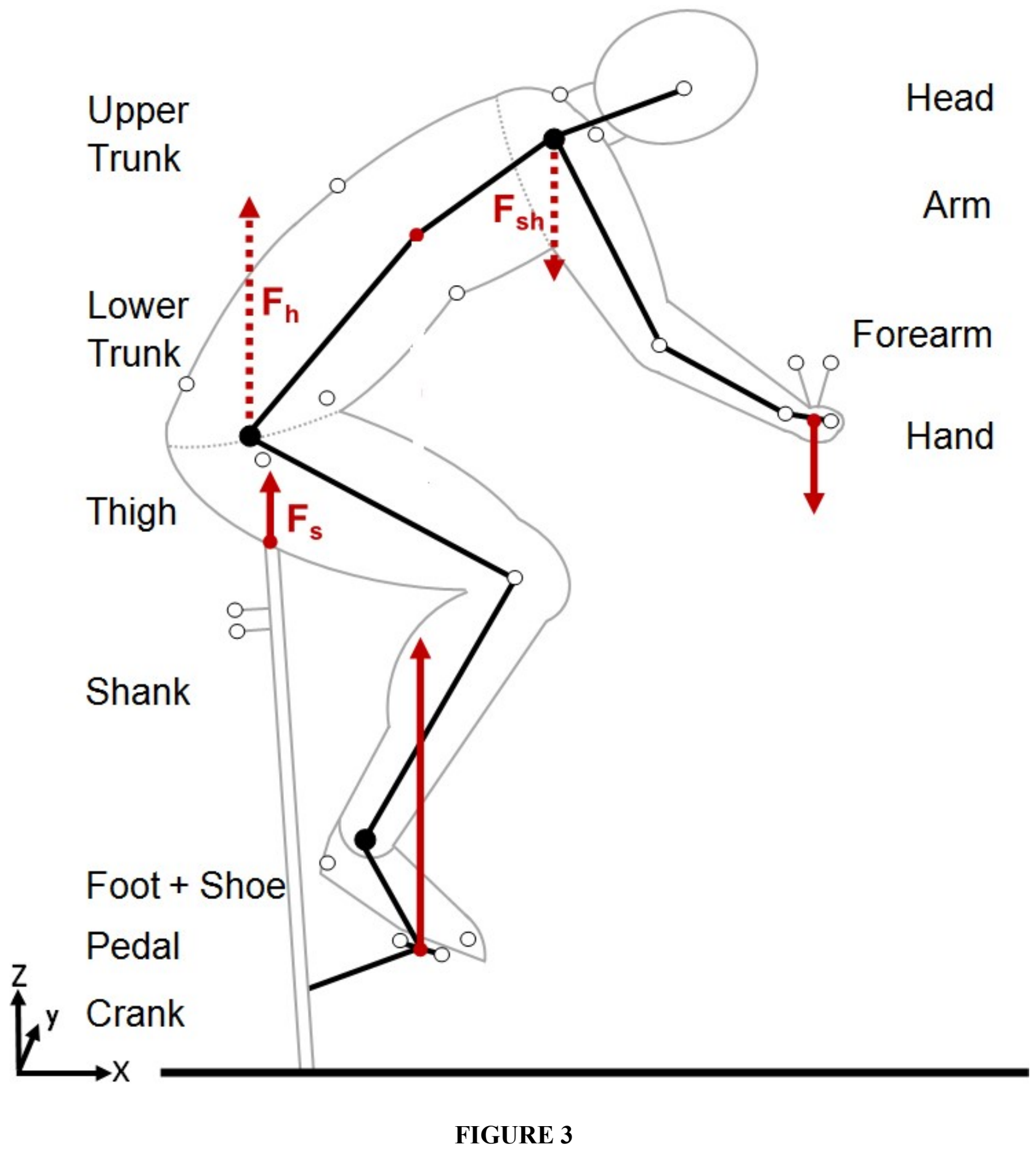




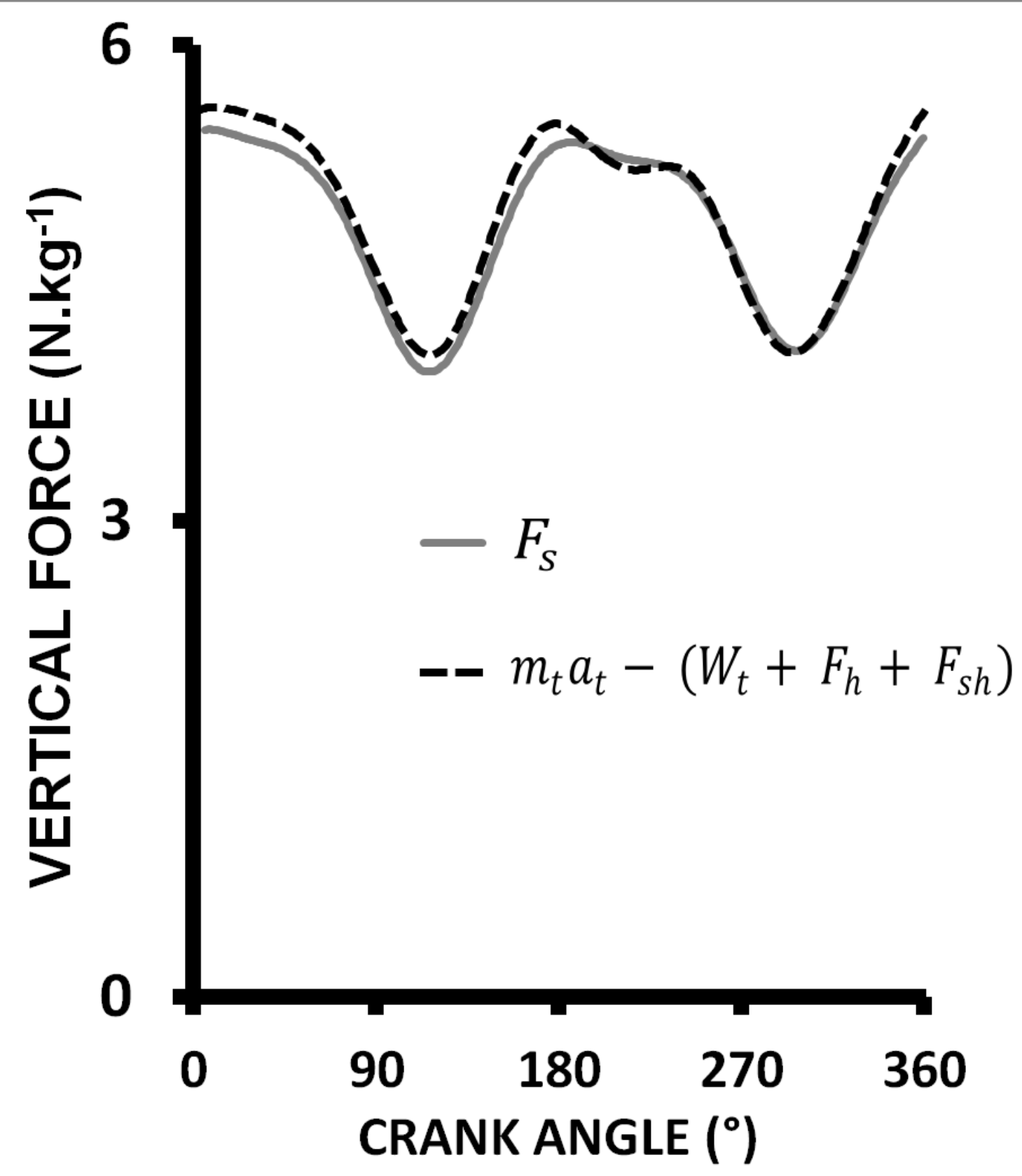

FIGURE 4 

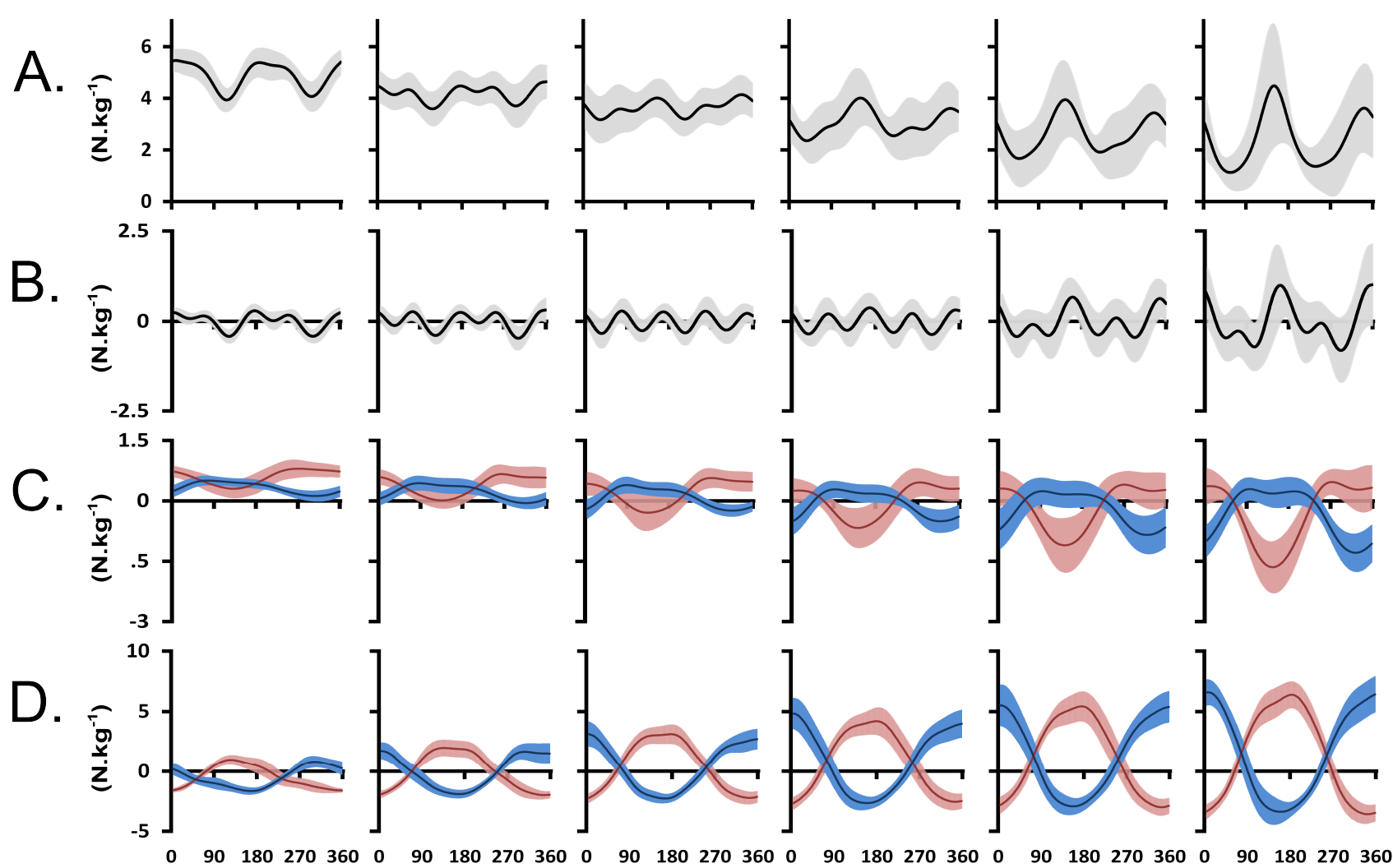

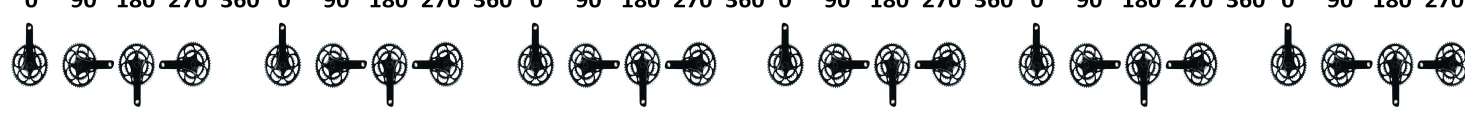

\begin{tabular}{|c|c|c|c|c|}
\hline & $40 \%$ & $60 \%$ & $80 \%$ & $100 \%$ \\
\hline
\end{tabular}

FIGURE 5

539 


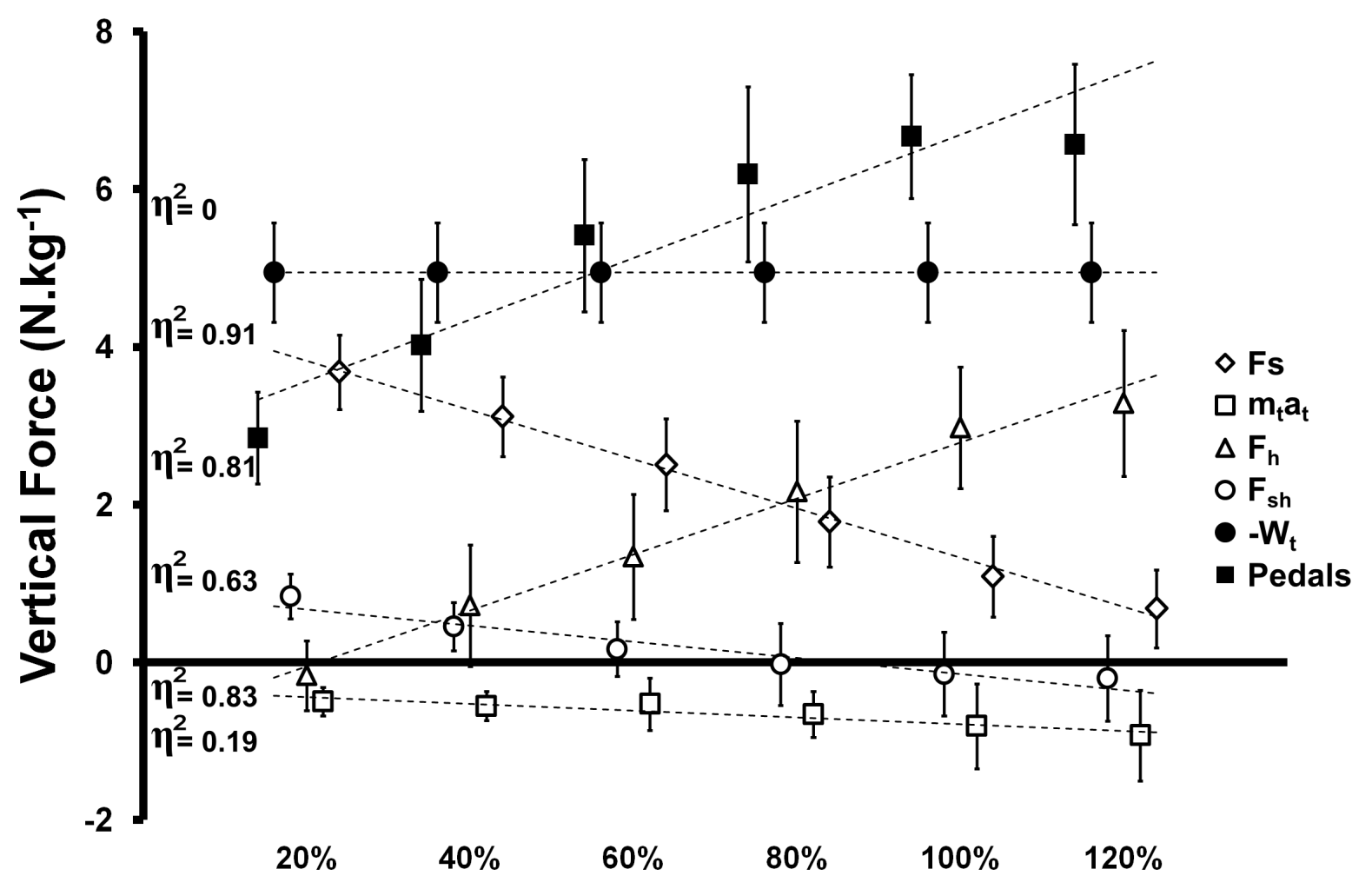

FIGURE 6 